\title{
Neutrophil protease 3 is present in human atherosclerotic lesions and modifies LDL and HDL particles
}

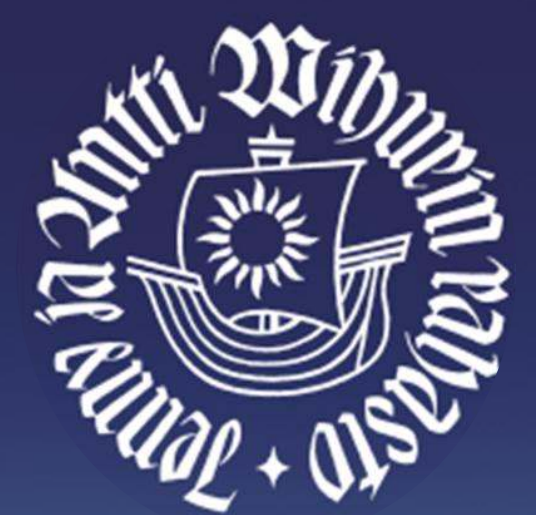

\author{
Su Duy Nguyen ${ }^{1}$, Katariina Maaninka $^{1}$, Mikko I. Mäyränpää ${ }^{2}$, Katariina Nurmi ${ }^{1}$, Jari Metso ${ }^{3,4}$, \\ Matti Jauhiainen ${ }^{3,4}$, Petri T. Kovanen ${ }^{1}$, Katariina Öörni ${ }^{1}$ \\ ${ }^{1}$ Wihuri Research Institute, Haartmaninkatu 8, 00290 Helsinki, Finland \\ 2Pathology, University of Helsinki and Helsinki University Hospital, Helsinki, Finland \\ ${ }^{3}$ Minerva Foundation Institute for Medical Research, Biomedicum, Helsinki, Finland \\ ${ }^{4}$ National Institute for Health and Welfare, Helsinki, Finland
}

\section{Objective}

Protease 3 (PR3), a major neutrophil protease present in neutrophil azurophilic granules, is a multifunctional enzyme involved in the regulation of pro-inflammatory processes but its role in atherosclerosis remains unexplored. Here, we aim to identify, whether PR3 is present in human atherosclerotic lesions and to examine how PR3 affects structure and functions of LDL and HDL.

\section{Methods and Results}

1. PR3 is present in advanced atherosclerotic lesions of human coronary arteries

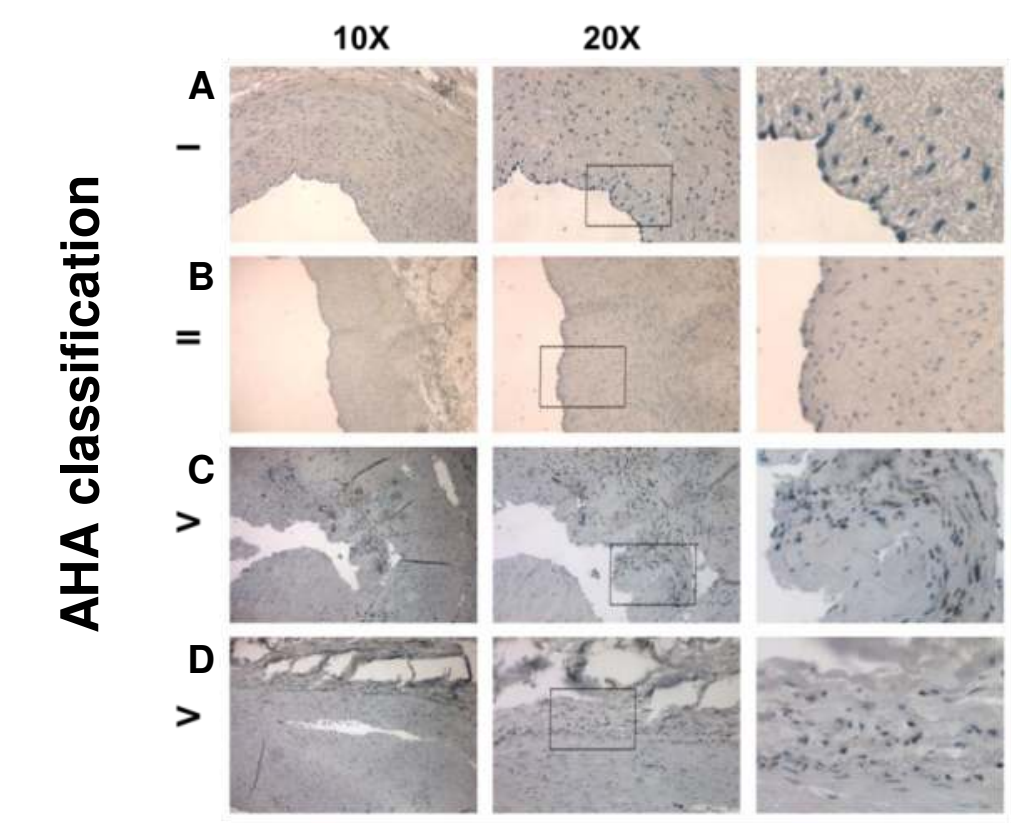

4. PR3-modified LDL binds strongly to

human aortic proteoglycans
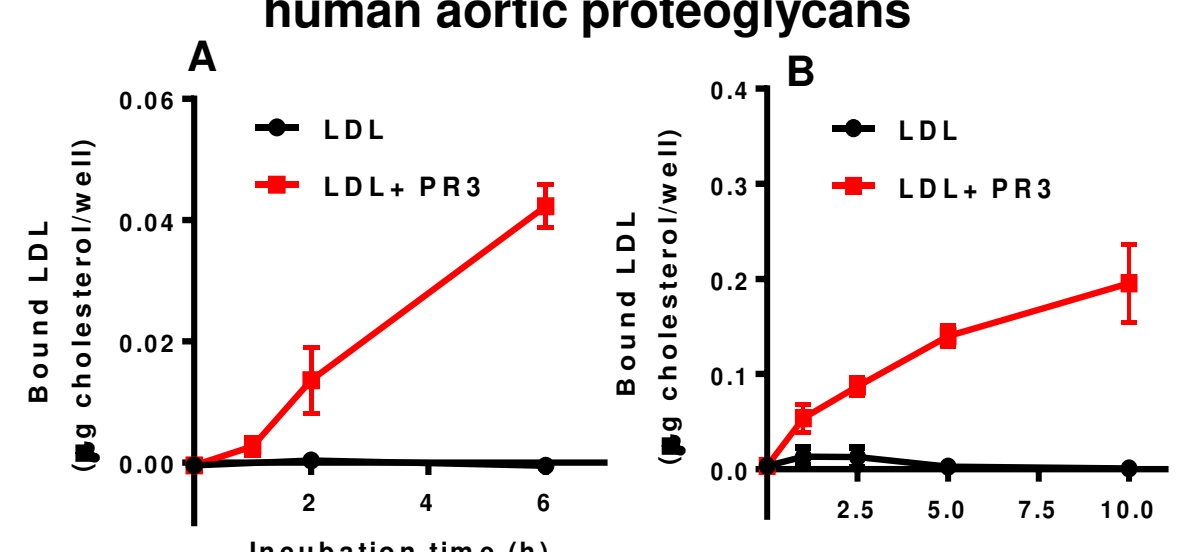

LDL protein (a/well)

Figure 4. A, LDL (1 $\mathrm{mg} / \mathrm{ml}$ ) was incubated with $60 \mu \mathrm{g} / \mathrm{ml}$ of PR3 for the indicated time, after which LDL and PR3-modified LDL $(2.5 \mu \mathrm{g} / \mathrm{ml})$ were added to microtiter wells precoated with human aortic proteoglycans. B, LDL $(1 \mathrm{mg} / \mathrm{ml})$ was incubated with $60 \mu \mathrm{g} / \mathrm{ml}$ of PR3 for 6h after which the aliquots of LDL and PR3-modified LDL (0-10 $\mu \mathrm{g} / \mathrm{ml}$ ) were added to microtiter wells precoated with human aortic proteoglycans. The amounts of proteoglycan-bound LDL were determined by measuring the amounts of cholesterol in the wells. As shown in $\mathbf{A}$ and $\mathbf{B}$, treatment of LDL with PR3 strongly enhanced the binding of the LDL to human aortic proteoglycans in a time- and dosedependent manner, respectively.

Figure 5. GM-CSF differentiated macrophages were incubated with medium (control), native LDL, PR3-treated LDL or acetylated LDL (50 $\mu \mathrm{g} / \mathrm{ml})$ for $24 \mathrm{~h}$ in DMEM. A, Cellular lipids were extracted with isopropanol: hexane and total cholesterol was determined and normalized to control. As shown, total cholesterol content was significantly increased in PR3-LDL-treated cells compared with control. B, The macrophage foam cells were stained with Oil Red $\mathrm{O}$. Scale bar $50 \mu \mathrm{m}$. C, Foam cell quantification of peritonea macrophages from studies described in $\mathbf{B}$. As shown in $\mathbf{B}$ and $\mathbf{C}$, increases in intracellular lipids were found in the macrophages incubated with PR3-LDL, indicating that modification of LDL by PR3 enhanced foam cell formation. ${ }^{*} p<0.05,{ }^{* *} p<0.01$.

\section{PR3 degrades apolipoproteins in mature sperical HDL}
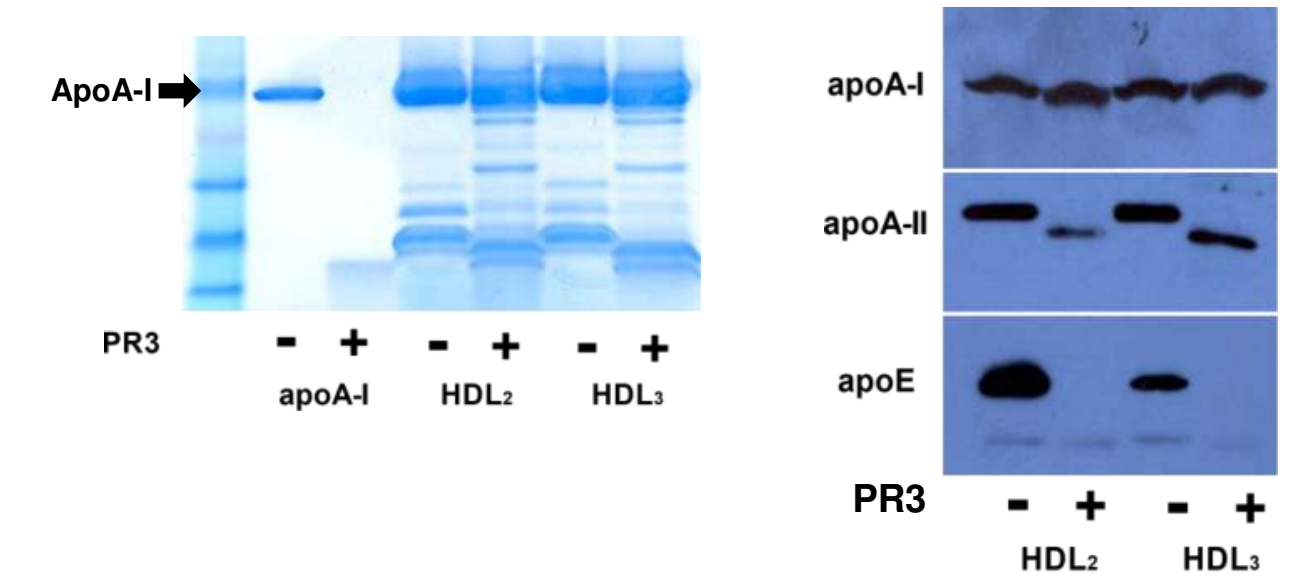

Figure 7. A, Spherical $\mathrm{HDL}_{2}$ or $\mathrm{HDL}_{3}(1 \mathrm{mg} / \mathrm{ml})$ was incubated with 25 $\mathrm{gg} / \mathrm{ml}$ of PR3 for $60 \mathrm{~min}$, and analyzed using 4-12\% gradient SDS-PAGE. B, Proteins in the HDL particles were immunoblotted with anti-human apoApolyclonal antibody, anti-human apoA-II monoclonal antibody or with antihuman apoE monoclonal antibody. Treatment of $\mathrm{HDL}_{2}$ and $\mathrm{HDL}_{3}$ with PR3 human apoE monoclonal antibody. Treatment of $\mathrm{HDL}_{2}$ and $\mathrm{HDL}_{3}$ with $\mathrm{PR} 3$
led to a significant change in apolipoprotein patterns. While apoA-1 associated with HDLs appeared to be degraded only a small extent, apoA-II and ApoE were fully degraded.

\section{PR3 induces rapid degradation of apoB-100 in LDL}

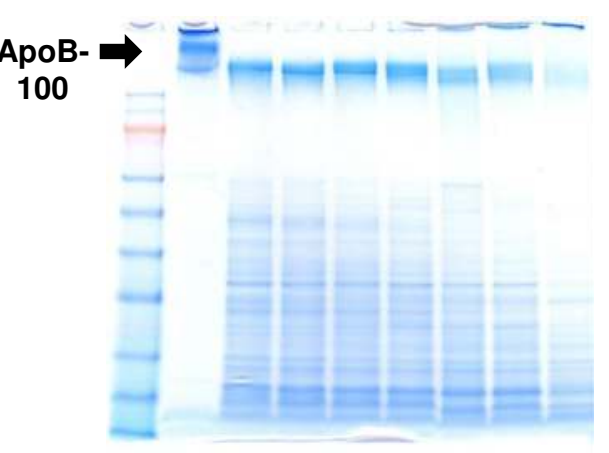

time (h) $00.250 .5122 \quad 4 \quad 624$

Figure 2. LDL $(1 \mathrm{mg} / \mathrm{ml})$ was incubated with $50 \mu \mathrm{g} / \mathrm{ml}$ of PR3 for the indicated time, and the change in apoB-100 structure was analyzed using $4-12 \%$ gradient SDS-PAGE. As shown, PR3 rapidly degraded apoB-100 within 15 min incubation, indicating that apoB100 in LDL is highly susceptible to PR3.

\section{Modification of LDL by PR3 induces foam cell formation}

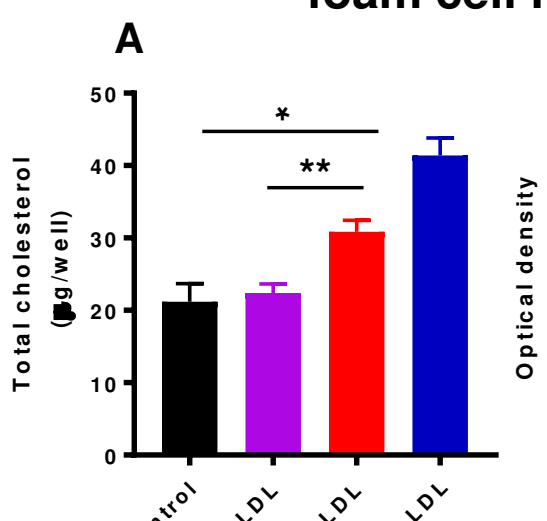
C

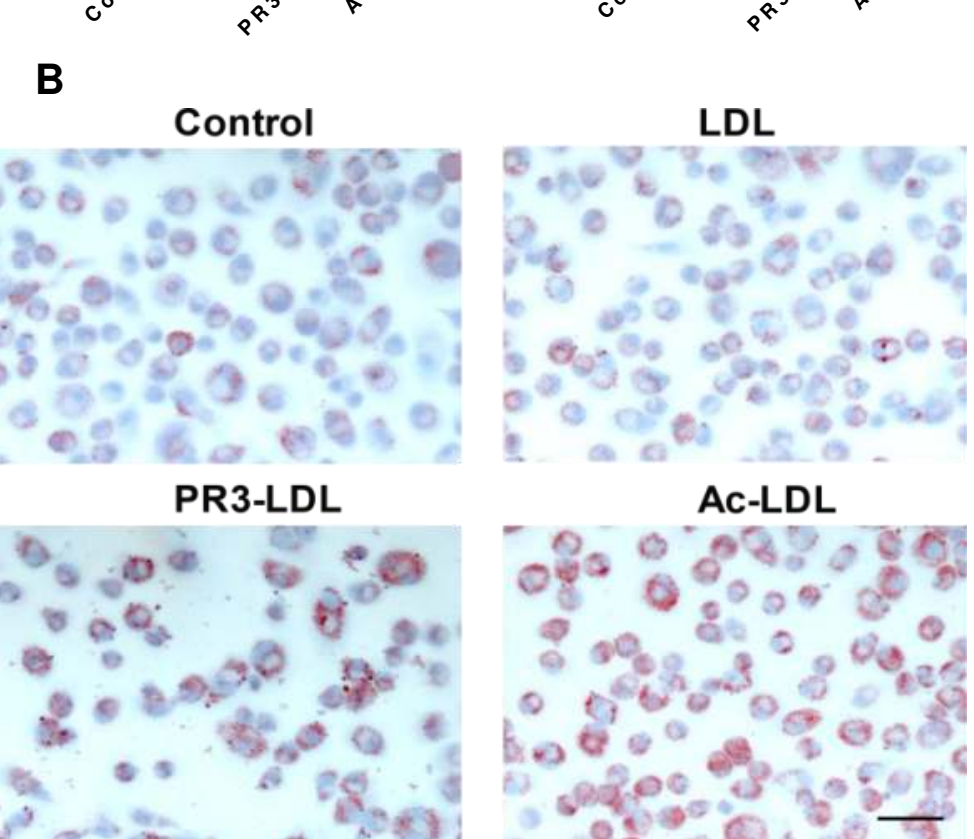

8. PR3 reduces the ability of apoA-I and HDL to promote cholestetol efflux

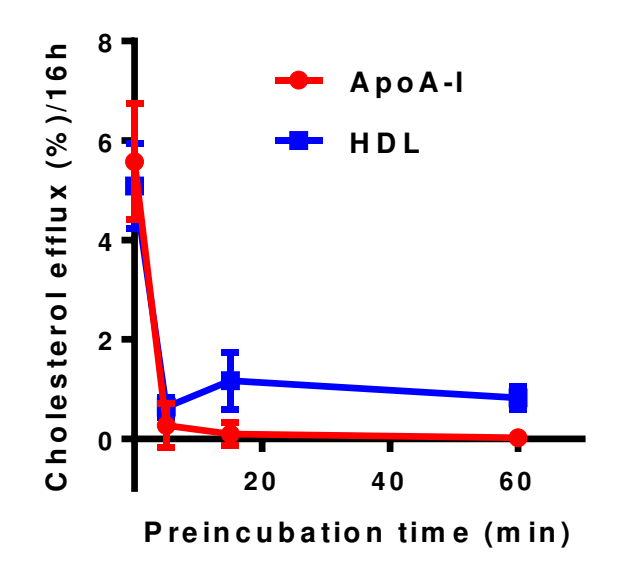

Figure 8. ApoA-I or total HDL (1 mg/ml) was preincubated with $25 \mu \mathrm{g} / \mathrm{ml}$ of PR3 for the indicated time. Foam cell formation was induced by incubation of GM-Macrophages with 25 $\mu \mathrm{g} / \mathrm{ml}$ of $\left[{ }^{3} \mathrm{H}\right]-\mathrm{CE}$-acetyl-LDL for $24 \mathrm{~h}$. GM-Mac- derived foam cells were then incubated with apoA-I, PR3-treated apoA-I $(10 \mu \mathrm{g} / \mathrm{ml})$, HDL or PR3-treated HDL $(25 \mu \mathrm{g} / \mathrm{ml})$ for $16 \mathrm{~h}$, the media were collected, centrifuged to remove cellular debris, and the radioactivity of each supernatant was determined by liquid scintillation counting. Cells were solubilized, and radioactivity was determined in the cell lysates. Cholesterol efflux was calculated as $\mathrm{dpm}_{\text {medium }} /\left(\mathrm{dpm}_{\text {cells }}+\mathrm{dpm}_{\text {medium }}\right) \times 100$. As shown, pretreatment with PR3 for only 5 min resulted in complete loss of cholesterol efflux by apoA-I into the medium. Furthermore, pretreatment of HDL with PR3 for 5 min led almost complete loss of its ability to promote cholesterol efflux.

\section{Conclusion}

The present data demonstrate that PR3 is a novel protease found in human advanced atherosclerotic lesions, and suggest that PR3 may have a role in the development of atherosclerosis by modifying both LDL and HDL particles. 